\title{
Adiponectin downregulation is associated with volume overload-induced myocyte dysfunction in rats
}

\author{
Li-li WANG ${ }^{1}$, Dori MILLER ${ }^{1}$, Desiree WANDERS ${ }^{1}$, Gayani NANAYAKKARA ${ }^{2}$, Rajesh AMIN², Robert JUDD ${ }^{1}$, Edward E MORRI- \\ SON ${ }^{1}$, Ju-ming $\mathrm{ZHONG}^{1, *}$
}

${ }^{1}$ Department of Anatomy, Physiology and Pharmacology, College of Veterinary Medicine, ${ }^{2}$ Department of Drug Discovery and Development, Harrison School of Pharmacy, Auburn University, Alabama, USA

\begin{abstract}
Aim: Adiponectin has been reported to exert protective effects during pathological ventricular remodeling, but the role of adiponectin in volume overload-induced heart failure remains unclear. In this study we investigated the effect of adiponectin on cardiac myocyte contractile dysfunction following volume overload in rats.

Methods: Volume overload was surgically induced in rats by infrarenal aorta-vena cava fistula. The rats were intravenously administered adenoviral adiponectin at 2-, 6- and 9-weeks following fistula. The protein expression of adiponectin, adiponectin receptors (AdipoR1/R2 and T-cadherin) and AMPK activity were measured using Western blot analyses. Isolated ventricular myocytes were prepared

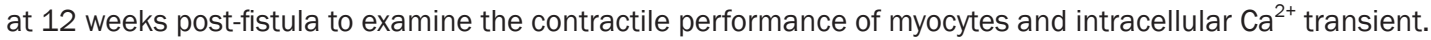

Results: A-V fistula resulted in significant reductions in serum and myocardial adiponectin levels, myocardial adiponectin receptor (AdipoR1/R2 and T-cadherin) levels, as well as myocardial AMPK activity. Consistent with these changes, the isolated myocytes exhibited significant depression in cell shortening and intracellular $\mathrm{Ca}^{2+}$ transient. Administration of adenoviral adiponectin significantly increased serum adiponectin levels and prevented myocyte contractile dysfunction in fistula rats. Furthermore, pretreatment of isolated myocytes with recombinant adiponectin $(2.5 \mu \mathrm{g} / \mathrm{mL})$ significantly improved their contractile performance in fistula rats, but had no effects in control or adenoviral adiponectin-administered rats.

Conclusion: These results demonstrate a positive correlation between adiponectin downregulation and volume overload-induced ventricular remodeling. Adiponectin plays a protective role in volume overload-induced heart failure.
\end{abstract}

Keywords: congestive heart failure; adiponectin; volume overload; myocyte contractility; calcium transient

Acta Pharmacologica Sinica (2016) 37: 187-195; doi: 10.1038/aps.2015.84; published online 30 Nov 2015

\section{Introduction}

The death rate attributable to congestive heart failure (CHF) has declined, but the high prevalence of disease remains an issue in the United States ${ }^{[1]}$. Mechanical stimuli in the form of pressure overload and volume overload are believed to be the major driving forces that initiate cardiac remodeling and increase the risk for development of CHF and sudden death $^{[2]}$. Pressure overload is often caused by high blood pressure and aortic stenosis. Volume overload is usually induced by valvular regurgitation, chronic myocardial infarction and pregnancy. The molecular mechanisms underlying pressure overload typically induces concentric hypertrophy, while volume overload increases diastolic load and results in eccentric hypertrophy characterized by sarcomere replication in

\footnotetext{
* To whom correspondence should be addressed. E-mail zhongju@auburn.edu

Received 2015-06-29 Accepted 2015-08-26
}

series and longitudinal cell growth. This leads to an increase in ventricular volume with little increase in wall thickness ${ }^{[3]}$. Although pressure overload- and myocardial infarctioninduced cardiac hypertrophy have been well studied, there is far less known about the mechanisms causing volume overload-induced heart failure, even though it is a clinically common cause of CHF. Recent animal studies demonstrated that cardiac remodeling secondary to volume overload is closely related to ATP starvation and metabolic abnormalities in myocardium, which further exacerbates the development of heart failure after volume overload ${ }^{[4,5]}$. Using a fistula rat model, Wang et $a l^{[6]}$ observed that compensated hypertrophy occurred between 2 and 8 week post fistula with normal or mildly depressed hemodynamic function. Heart failure occurred subsequently between 8 to 16 weeks with depressed in vivo and in vitro LV systolic pressure. Previous study from our laboratory indicates that depressed ventricular contractility is intrinsic to the individual myocytes ${ }^{[7]}$. In that study the in vivo cardiac 
size and performance following fistula surgery was evaluated by M-mode echocardiography. A progressive increase in LV end diastolic and end systolic diameters was observed in fistula rats at 5-week and 10-week following surgery, indicating a developmental ventricular dilatation. Associated with ventricular dilatation, in vivo LV fractional shortening was lower in 5-week fistula rats and further reduced in 10-week fistula rats. Although there was no significant difference in body weights between AV fistula rats and age-matched sham-operated control rats, a progressive increase of heart weight and heart/body ratio was observed in fistula rats. In the same study rat ventricular myocytes following sustained volume overload demonstrated significant depression in cell shortening, which was associated with reduced peak intracellular $\mathrm{Ca}^{2+}\left(\left[\mathrm{Ca}^{2+}\right]_{\mathrm{i}}\right)$ transients. Abnormal myocyte contractility and $\left[\mathrm{Ca}^{2+}\right]_{i}$ in these cells resulted from the deficiency of the key Ca handling proteins including SR $\mathrm{Ca}^{2+}$ ATPase and ryanodine receptors ${ }^{[7]}$. However, the mechanism of volume overload leading to the derangement of energy metabolism in myocardium is not clear.

Adiponectin (APN) is collagen-like protein hormone circulating at a very high concentration in the range from 3 to $30 \mu \mathrm{g} / \mathrm{mL}$, thus accounting for $0.01 \%$ of total plasma protein $^{[8,9]}$. Initially, APN was believed to be exclusively expressed by white adipose tissue, but recent evidence indicates that myocardium can locally produce bioactive APN. Ding et al ${ }^{[10]}$ demonstrated that APN and both APN receptors are expressed in adult ventricular cardiomyocytes and upregulated by activation of peroxisome proliferator-activated receptor gamma. In myocardium, APN functions to modulate glucose uptake and fatty acid oxidation via AMP-activated protein kinase (AMPK)-dependent signaling pathways ${ }^{[11,12]}$. These physiological effects of APN are mediated via interactions with its cell-surface receptors: AdipoR1, AdipoR2 and T-cadherin. The level of circulating APN is a predictor for the development of heart failure. Low APN level in serum (hypoadiponectinemia) is associated with cardiac hypertrophy and impairments of cardiac function under pathological conditions ${ }^{[13-16]}$. APN deficiency in APN-knockout mice further exacerbates cardiac dysfunction following pressure overload induced cardiac remodeling ${ }^{[17,18]}$. Conversely, supplementation of APN suppresses pathologic cardiac remodeling and improves cardiac function, thus protecting heart function against myocardial injury induced by pressure overload ${ }^{[18,19]}$. However, no study has addressed the question that whether APN is beneficial or deleterious for the development of heart failure secondary to volume overload. Therefore, the aim of this study is to examine the effects of APN on the volume overload-induced heart failure in rats. Since our previous study indicates that depressed ventricular contractility is intrinsic to the individual myocytes ${ }^{[7]}$, the focus of the present study was evaluating the potential protective roles of APN on the isolated myocyte contractility.

\section{Materials and methods}

\section{Surgical preparation and APN administration}

The animal use procedures were performed conforming the
$\mathrm{NIH}$ guidelines, and the animal use protocol was approved by the Auburn University Institutional Animal Care and Use Committee. Male Sprague Dawley rats (250-300 g, 8 weeks) were randomly divided into three groups: Control $(n=6)$, Fistula $(n=7)$, and Fistula+Ad-APN (adenoviral adiponectin, Ad-APN; $n=6)$. Chronic volume overload was induced through infrarenal aorto-caval (AV) fistula ${ }^{[7]}$. Infrarenal aortavena $(\mathrm{AV})$ fistula was performed on rats under $\mathrm{O}_{2}$-isofluorane inhalation anesthesia (2\%). A midline abdominal incision was performed and the abdominal aorta and inferior vena cava were exposed. An 18-gauge needle was inserted into the exposed abdominal aorta and advanced through the medial wall into the vena cava to create a fistula. The needle was withdrawn, and the aortic puncture site was sealed by cyanoacrylate glue. Fistula patency was visually confirmed by the pulsatile flow of oxygenated blood into the vena cava. Abdominal musculature and skin incisions were sutured with $3 / 0$ catgut and autoclip. Control animals underwent similar procedure except that no aortic puncture was applied.

Fistula + Ad-APN rats received $2 \times 10^{8}$ plaque-forming units of Ad-APN at 2-, 6- and 9 weeks by intravenous injection via the tail vein. Ad-APN was obtained from Eton Biosciences (SanDiego, CA, USA) (Cat \# 0100182001) and stored an $-80^{\circ} \mathrm{C}$. Ad-APN was thawed and diluted to the right concentration by saline before injection.

\section{Analysis of total serum APN levels}

Blood samples were collected from tail vein at 3 days before, 5 weeks and 10 weeks after fistula surgery. Serum was isolated by centrifugation and preserved at $-80^{\circ} \mathrm{C}$ until analysis. Total serum APN levels were determined by rat adiponectin ELISA kit (Millipore Co, Billerica, CA, USA).

\section{Ventricle myocyte isolation}

At 12 weeks post-fistula, ventricular myocytes were isolated as previously described ${ }^{[7]}$. In brief, rat was injected ip of Fetal Plus $0.5 \mathrm{~mL}$, and decapitated under deep anesthesia. The heart was quickly removed and perfused with oxygenated $\mathrm{Ca}^{2+}$-free Krebs-Henseleit (KH) buffer $(118 \mathrm{mmol} / \mathrm{L} \mathrm{NaCl}, 4.8$ $\mathrm{mmol} / \mathrm{L} \mathrm{KCl}, 25 \mathrm{mmol} / \mathrm{L}$ HEPES, $1.25 \mathrm{mmol} / \mathrm{L} \mathrm{K}_{2} \mathrm{HPO}_{4}, 1.25$ $\mathrm{mmol} / \mathrm{L} \mathrm{MgSO}_{4}, 11 \mathrm{mmol} / \mathrm{L}$ glucose, $\mathrm{pH}$ at 7.4) for $5 \mathrm{~min}$, followed with $\mathrm{KH}$ buffer containing $5 \mathrm{mmol} / \mathrm{L} \mathrm{BDM}, 2 \mathrm{mmol} / \mathrm{L}$ carnitine, $5 \mathrm{mmol} / \mathrm{L}$ taurine, $2 \mathrm{mmol} / \mathrm{L}$ glutamic acid, $0.045 \%$ collagenase (Worthington; Type II, $371 \mathrm{U} / \mathrm{mg}$ ), and $20 \mu \mathrm{mol} / \mathrm{L}$ $\mathrm{CaCl}_{2}$ for approximately $15-18 \mathrm{~min}$. Ventricles were then isolated and mechanically dispersed in $0.02 \mathrm{mmol} / \mathrm{L} \mathrm{Ca}^{2+}$ Kraftbrühe (KB) solution, filtered and centrifuged at $15 \times g$ for 5 $\mathrm{min}$ at room temperature. Isolated cardiomyocytes were then resuspended in $\mathrm{KH}$ buffer with gradually increasing concentrations of $\mathrm{Ca}^{2+}$ to yield $\mathrm{Ca}^{2+}$-tolerant cells.

Measurement of myocyte contractility and intracellular $\mathrm{Ca}^{2+}$ transient Ventricular myocytes were collected for measurement of contractility and intracellular $\mathrm{Ca}^{2+}$ transient using fluorescence and edge-detecting system (IonOptix, Milton, MA, USA) ${ }^{[20]}$. Cardiomyocytes were incubated with $2.5 \mu \mathrm{mol} / \mathrm{L}$ Fura-2/AM 
at room temperature for $20 \mathrm{~min}$. Cells were then washed in $1.8 \mathrm{mmol} / \mathrm{L} \mathrm{Ca}^{2+} \mathrm{KH}$ buffer to remove excess Fura-2. Fura-2loaded cardiomyocytes were mounted on an inverted microscope (Nikon TE, 2000, Tokyo, Japan) and perfused with 1.8 $\mathrm{mmol} / \mathrm{L} \mathrm{Ca}^{2+} \mathrm{KH}$ buffer by gravity $(\sim 2 \mathrm{~mL} / \mathrm{min})$ at room temperature. Only rod shaped, clearly striated, and mechanically quiescent myocytes were chosen for the study. Myocyte contraction was elicited at $0.5 \mathrm{~Hz}$ frequency by field stimulation. The Fura-2-fluorescence was excited by collimated light beam from a $150 \mathrm{~W}$ Xe arc lamp. The intracellular $\mathrm{Ca}^{2+}$ transient was recorded by $340 / 380 \mathrm{~nm}$ ratio excitation paired with an emission wavelength at $510 \mathrm{~nm}$. The contractile function of ventricular myocytes was determined by percent change of the sarcomere shortening simultaneously with intracellular $\mathrm{Ca}^{2+}$ transients. In some experiments, ventricular myocytes isolated from different groups were incubated with $2.5 \mu \mathrm{g} / \mathrm{mL}$ recombinant APN for $2 \mathrm{~h}$ in $1.8 \mathrm{mmol} / \mathrm{L} \mathrm{Ca}^{2+} \mathrm{KH}$ buffer.

\section{Western blot}

Cell lysates were prepared from myocytes isolated from rats in each group. The total protein contents were determined by the Bradford method. Equal amounts of protein for each sample were resolved by $10 \%$ SDS-PAGE gel and subjected to Western blot. The membrane was incubated with primary antibody against target proteins followed by incubation with IRDye Infrared Dyes (Li-Cor, Lincoln, NE, USA). Bands were imaged and quantified using the Odyssey Infrared Imaging System (Li-Cor, Lincoln, NE, USA). The target proteins were normalized to $\beta$-Actin or GAPDH.

\section{Materials}

Recombinant adiponectin was purchased from Phoenix Pharmaceuticals, Inc (Burlingame, CA). APN antibody was from Abcam, Inc (Cambridge, MA, USA). AdipoR1, AdipoR2 and T-cadherin antibodies were from Santa Cruz Biotechnology, Inc (Santa Cruz, CA, USA). Phospho-AMPKa (Thr172) and AMPKa antibodies were purchased from Cell Signaling (Danvers, MA, USA). His-tag adiponectin recombinant adenovirus was from Eton Bioscience Inc (San Diego, CA, USA). IRDye secondary antibodies were from Li-Cor (Lincoln, NE, USA). All chemicals were analytical grade and were from SigmaAldrich (St Louis, USA).

\section{Statistical analysis}

Myocyte contraction and intracellular $\mathrm{Ca}^{2+}$ transient were performed on five to seven myocytes randomly chosen from each animal in each group and data averaged to represent that animal. Values were expressed as mean \pm SEM and $n$ as the number of animals studied. Data from the different groups of animals were compared using two-tailed unpaired Student's $t$ test, and one-way ANOVA with a Student-Newman-Kuels post-test, whenever appropriate. $P$ value of $<0.05$ was considered to be significantly different.

\section{Results}

To clarify whether circulating APN levels are inversely cor- related with the progression of heart failure induced by AV fistula, ELISA assay was applied to measure serum total APN levels in all groups of rats at 3 days before, 5 weeks and 10 weeks after fistula. At 3 days before fistula, no significant difference in serum APN levels was observed between rats from different groups (data not shown). At 5 weeks and 10 weeks post-fistula, serum APN levels were significantly reduced in fistula rats. In vivo administration of Ad-APN significantly increased the total APN levels in serum at 5 weeks and 10 weeks post-fistula, as compared with control and fistula rats (Figure 1A).

Similar to the reduction of serum APN levels, the protein expression of total APN was significantly reduced in ventricular myocytes isolated from fistula rats at 12 weeks post-fistula. However, Ad-APN administration markedly prevented the reduction of APN levels in myocytes. No significant difference was observed in the myocardial protein APN levels between fistula+Ad-APN and control rats (Figure 1B).
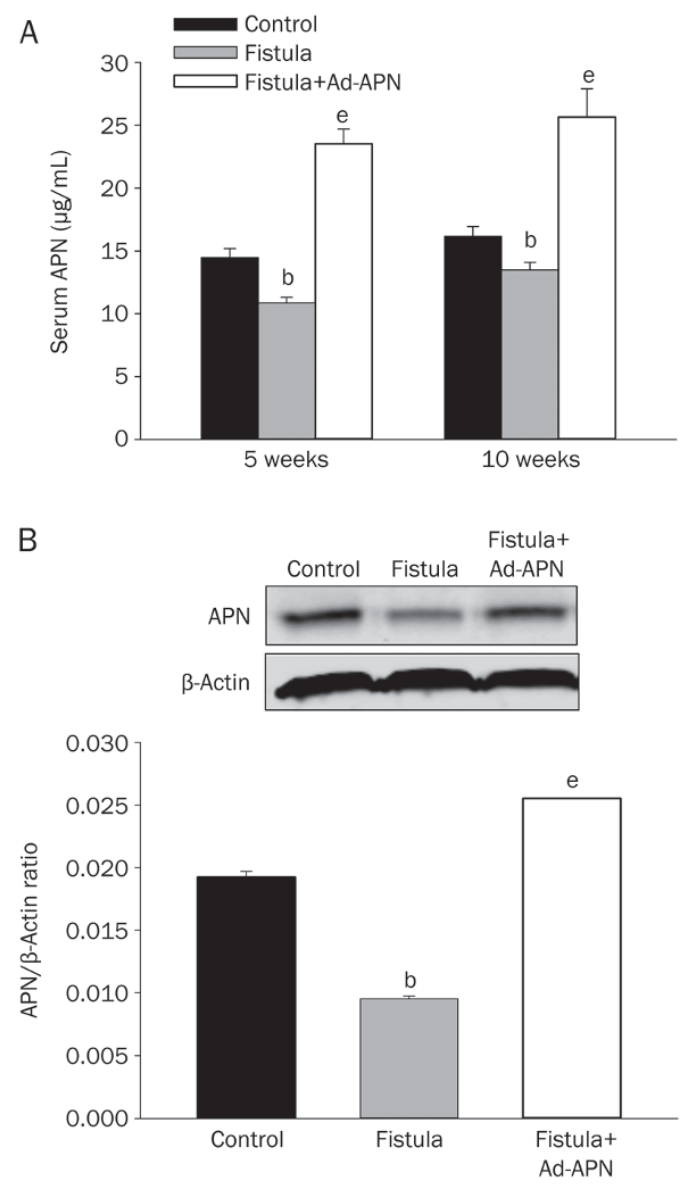

Figure 1. Total adiponectin (APN) levels following fistula. (A) Serum total APN levels at 5 weeks and 10 weeks post-fistula. (B) Myocardial protein expression of APN normalized to $\beta$-actin at 12 weeks post-fistula with or without the administration of adenovirus-APN (Ad-APN). Results were presented as mean \pm SEM for control $(n=6)$, fistula $(n=7)$ and fistula+AdAPN $(n=6) .{ }^{b} P<0.05$ compared with control. ${ }^{e} P<0.05$ compared with Fistula. 
AdipoR1 and AdipoR2 serve as predominant APN receptors and mediate glucose uptake and fatty acid oxidation in myocytes $^{[21]}$. In addition to AdipoR1 and AdipoR2, T-cadherin has also been identified as a membrane receptor for hexameric and high-molecular-weight forms of APN, which is also critical for APN-dependent energy metabolism in myocytes ${ }^{[22,23]}$. Western blot analysis was applied to determine the protein levels of AdipoR1/R2 and T-cadherin in isolated ventricular myocytes at 12 weeks post-fistula. Results demonstrated that myocardial protein expressions of AdipoR1/R2 and T-cadherin in the Fistula group of rats were decreased at 12 weeks post-fistula as compared with control. Conversely, Ad-APN treatment prevented the reduction of receptor proteins. Myocardial protein expression of AdipoR1/R2 and T-cadherin in myocytes isolated from fistula+Ad-APN rats was comparable to that in myocytes from control rats (Figure 2).

APN functions to mediate myocardial glucose uptake and fatty acid oxidation as well as to provide cardioprotective roles by increased AMPK activity through phosphorylation of AMPK-a (Thr172) ${ }^{[24]}$. In this study, Western blot was used to assess the phosphorylation status of AMPK at threonine residue 172 of the a subunit in myocytes from rats in each group. At 12 weeks post-fistula, AMPK-a phosphorylation was significantly decreased, while the total AMPK level was similar in fistula myocytes when compared to control. However, AdAPN treatment significantly improved AMPK-a phosphorylation in myocytes from fistula+Ad-APN rats compared with fistula myocytes. There was no significant difference of AMPK-a phosphorylation in myocytes between fistula+Ad-APN and control rats (Figure 3 ).

To investigate the effects of APN on myocyte contractile performance when subjected to volume overload, we compared the myocyte shortening among different groups of animals. The resting sarcomere lengths were comparable among different groups of myocytes. When myocytes were electri-
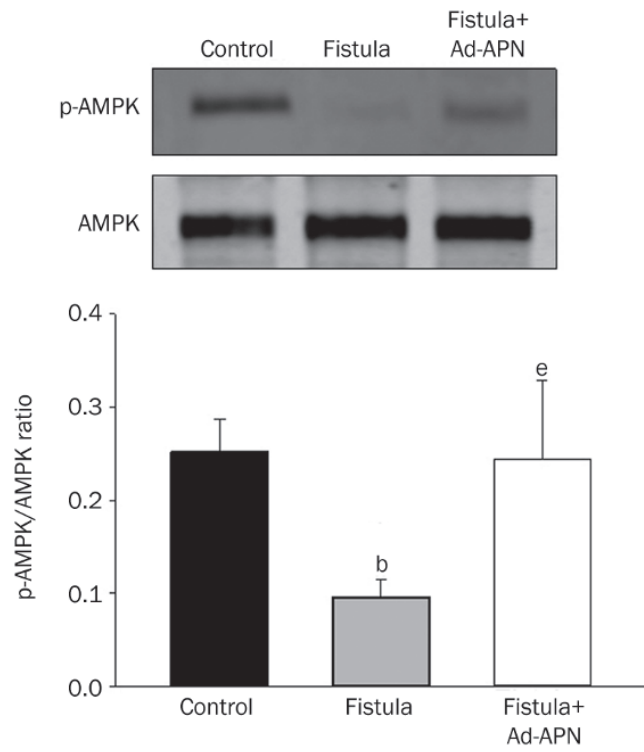

Figure 3. AMP-activated protein kinase (AMPK) phosphorylation at 12 weeks post-fistula. (A) Representative Western blot of phospho-AMPK $\alpha$ (Thr172) and AMPK $\alpha$ in isolated ventricular myocytes. (B) PhosphoAMPK $\alpha($ Thr172) normalized to AMPK $\alpha$. Results were presented as mean \pm SEM for control $(n=6)$, fistula $(n=7)$ and fistula+Ad-APN $(n=6)$. ${ }^{\mathrm{b}} P<0.05$ compared with control. ${ }^{\mathrm{e}} P<0.05$ compared with Fistula.

cally stimulated at $0.5 \mathrm{~Hz}$, mechanical properties showed that contractile performance was depressed in myocytes from Fistula rats as compared with control at 12 weeks post-fistula. Sarcomere shortening was significantly decreased in myocytes isolated from 12-week fistula rats. Similarly, fistula myocytes had a delayed relaxation as shown by the prolonged sarcomere relengthening when compared with that of control. In vivo Ad-APN administration prevented normal contractile dysfunction induced by fistula, and the contractile perfor-
A
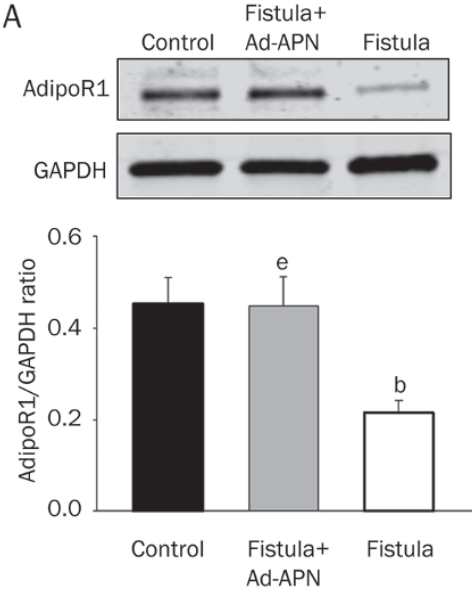

B
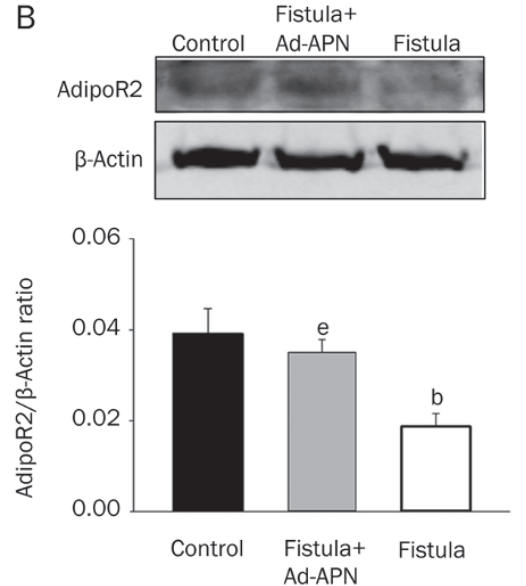

C
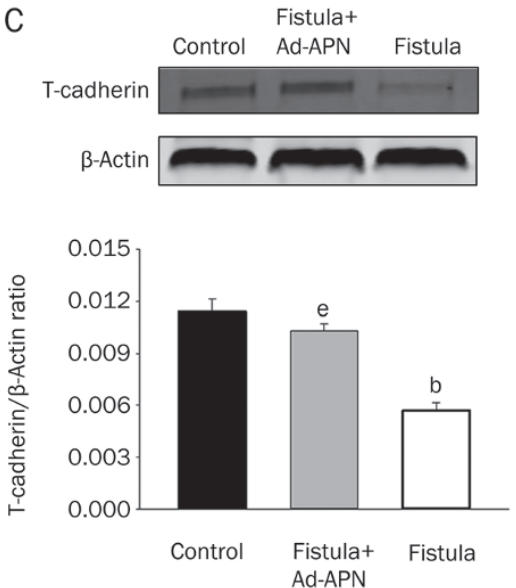

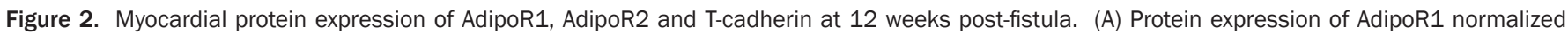

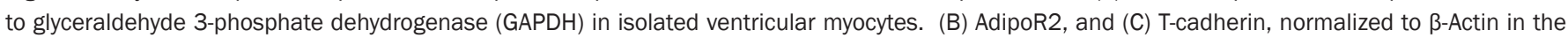

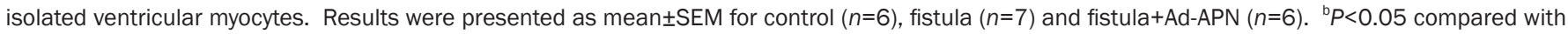
control. ${ }^{\mathrm{e}} \mathrm{P}<0.05$ compared with Fistula. 

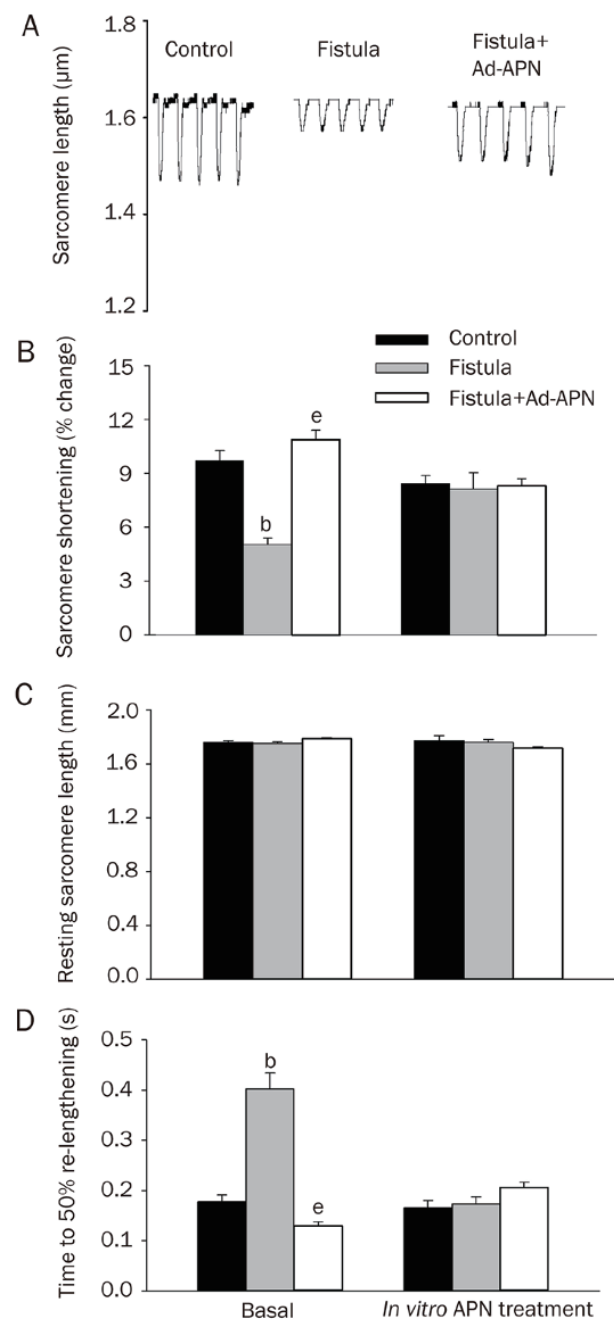

Figure 4. Contractile performance of ventricular myocytes with or without $2-4 \mathrm{~h}$ in vitro APN treatment $(2.5 \mu \mathrm{g} / \mathrm{mL})$. (A) Representative sarcomere length recordings from a control, a fistula and a fistula+Ad-APN myocytes. (B) Percent change of sarcomere shortening. (C) Resting sarcomere length. (D) Time to $50 \%$ sarcomere relengthening. Results were presented as mean \pm SEM for control $(n=6)$, fistula $(n=7)$ and fistula+AdAPN $(n=6) .{ }^{b} P<0.05$ compared with control. ${ }^{e} P<0.05$ compared with Fistula.

mance of myocytes from fistula+Ad-APN rats was not significantly different from the control myocytes (Figure 4).

To further examine the effects of APN on the myocyte performance at cellular levels, myocytes isolated from different groups of rats were treated with $2.5 \mu \mathrm{g} / \mathrm{mL}$ recombinant APN for 2-4 h before mechanical study. Pretreatment with recombinant APN significantly augmented the contractility of fistula myocytes and brought the cell shortening to the normal levels of control myocytes. On the other hand, recombinant APN treatment did not affect the contractile performance in myocytes from fistula+Ad-APN and control rats (Figure 4).

Consistent with contractile properties, intracellular $\mathrm{Ca}^{2+}$ transient was also depressed in fistula myocytes. Resting
Fura-2 ratios were similar among different groups of myocytes. However, the peak Fura-2 ratio was significantly lower, and the time-to- $50 \%$ Fura-2 ratio decay was remarkably prolonged in fistula myocytes compared with control. Conversely, Ad-APN treatment retained intracellular $\mathrm{Ca}^{2+}$ transient in myocytes from fistula+Ad-APN rats (Figure 5). In vitro treatment of myocytes with recombinant $\mathrm{APN}$ restored the reduction of intracellular $\mathrm{Ca}^{2+}$ transient in fistula myocytes to normal values of control myocytes, but did not affect the $\mathrm{Ca}^{2+}$ transient in myocytes from control and fistula+Ad-APN rats (Figure 5).

\section{Discussion}

To our knowledge, our study is the first to demonstrate the inverse relationship between APN levels and the progres-
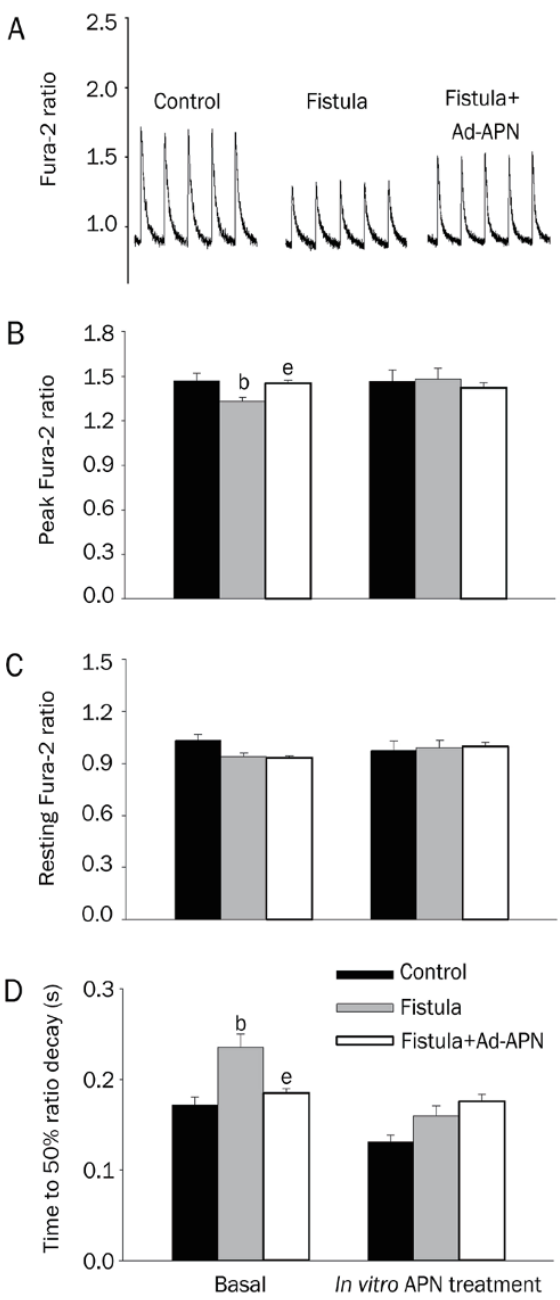

Figure 5. Intracellular $\mathrm{Ca}^{2+}$ transient in isolated ventricular myocyte with or without in vitro APN treatment $(2.5 \mu \mathrm{g} / \mathrm{mL})$. (A) Representative Fura-2 ratio $\left(F_{340 \mathrm{~nm}} / F_{380 \mathrm{~nm}}\right)$ recordings from a control, a fistula, and a fistula+Ad-APN myocytes. (B) Peak Fura-2 ratio $\left(F_{340 \mathrm{~nm}} / F_{380 \mathrm{~nm}}\right)$. (C) Resting Fura-2 ratio $\left(F_{340 \mathrm{~nm}} / F_{380 \mathrm{~nm}}\right)$. (D) Time to the $50 \%$ ratio decay (s). Results were presented as mean \pm SEM for control $(n=6)$, fistula $(n=7)$ and fistula+Ad-APN $(n=6)$. ${ }^{\mathrm{b}} P<0.05$ compared with control. ${ }^{\mathrm{e}} P<0.05$ compared with Fistula. 
sion of heart failure due to sustained volume overload. One of the key findings from this study is a progressive and significant reduction of APN levels in serum and ventricular myocytes following volume overload. In addition, protein expression of APN receptors were decreased in ventricular myocytes isolated from fistula rats at 12 weeks post-fistula, which was accompanied by reduced AMPK activation. Consistent with these findings, ventricular myocyte contractility and intracellular $\mathrm{Ca}^{2+}$ transients were markedly depressed at 12 weeks post-fistula. Conversely, in vivo administration of adenovirus-mediated overexpression of APN restored the APN levels, prevented the loss of protein expression of APN receptors, enhanced AMPK phosphorylation in myocytes from fistula+Ad-APN rats, and restored the contractile performance of ventricular myocytes. Moreover, in vitro treatment of ventricular myocytes from fistula rats with recombinant APN fully compensated the contractile deficiency, while treatment of recombinant APN does not have any effect on the contractile performance in myocytes isolated from control and fistula+Ad-APN rats.

Cardiac remodeling involves molecular, cellular and interstitial changes. Cardiac hypertrophy is a common type of cardiac remodeling following pathological stimulation and a major predictor of the development of heart failure ${ }^{[25]}$. Abnormal mechanical stimuli in the form of pressure overload and volume overload are believed to be the major driving forces that initiate cardiac remodeling and increase the risk for development of $\mathrm{CHF}$ and sudden death ${ }^{[2]}$. In response to pressure and volume stress, hypertrophic growth develops in two different ways: (1) concentric hypertrophy, due to pressure overload, is characterized by a relative increase in myocyte cross sectional area without a significant change in myocyte length. It leads to reduced ventricular volume and increased wall thickness; (2) eccentric hypertrophy, in response to volume overload, results in a relative increase in myocyte length without a significant change of cross section area. This causes dilatation and thinning of the heart wall ${ }^{[2,26]}$. At the molecular level, signaling analysis demonstrated striking differences in transducing signaling pathways that were activated under the different overload conditions. Pressure overload-induced cardiac hypertrophy is associated with increased activation of extracellular-signal-regulated kinases $1 / 2$ (ERK1/2) and persistent activation of $\mathrm{Ca}^{2+} /$ calmodulin-dependent protein kinase II (CaMKII) ${ }^{[25,27]}$. On the other hand, volume overload stimulates activation of the MEK5-ERK5 signaling branch of the greater mitogen-activated protein kinases (MAPKs) ${ }^{[25]}$ and Akt activation without much fibrosis signaling ${ }^{[27]}$. In addition, a total of 157 mRNAs and 13 microRNAs were differentially regulated in pressure-overload versus volume-overload mice models of heart failure ${ }^{[27]}$. In the present study, experimental data indicated a progressive and significant reduction of APN levels in serum and ventricular myocytes following volume overload. On the other hand, serum level of APN remained the same after pressure overload challenge ${ }^{[28]}$, while APN deficiency in APN knockout mice exacerbated cardiac dysfunction following pressure overload ${ }^{[1,17]}$. Taking together, our study further support the distinct phenotype differences between different mechanical stresses, indicating the requirement for specific pharmacological interventions for specific phenotype.

A previous study from our laboratory demonstrated an in vivo and in vitro progressive development of cardiac dysfunction following fistula surgery in rats ${ }^{[7]}$. The present study also demonstrated a progressive reduction of serum APN levels after fistula surgery. Moreover, reduction of APN was associated with depressed myocardial contractility and intracellular $\mathrm{Ca}^{2+}$ transient. Thus, hypoadiponectinemia may be a contributing factor to the progression of heart failure induced by volume overload. In accordance with this hypothesis, our data clearly showed that adenovirus-mediated APN overexpression prevented the volume overload-induced cardiac contractile function in fistula rats. Furthermore, in vitro treatment of fistula myocytes with APN fully recovered contractile dysfunction. These results suggested that APN offers cardiovascular protection in volume-overload cardiomyopathy.

APN is a protein hormone that functions to improve insulin sensitivity and modulate energy metabolism in tissues ${ }^{[2]}$. Different from most other adipocytokines, circulating APN levels are negatively correlated with body mass index ${ }^{[30]}$. Hypoadiponectinemia has been observed in obesity and its related diseases, including hypertension, type II diabetes and coronary heart disease ${ }^{[13-16,31]}$. Interestingly, although serum APN levels remain relatively stable in mice subjected to pressure overload $^{[28]}$. APN deficiency exacerbates cardiac dysfunction following pressure overload ${ }^{[11,32]}$. Conversely, APN supplementation prevents cardiac remodeling and ameliorates cardiac abnormalities due to pressure overload and myocardial infarction $^{[17,18]}$

APN is mainly expressed by adipocytes. However, cardiomyocytes are capable of synthesizing and secreting a small amount of $\mathrm{APN}^{[33,34]}$. Circulating APN seems to act as the predominant ligand for myocardial APN receptors and exert cardioprotective actions in an endocrine manner. Shibata et al described that APN accumulated in heart following ischemia-reperfusion injury through leakage from the vascular compartment and APN protein was only detectable in injured hearts but not sham-operated hearts ${ }^{[35]}$. However, recent studies found that the locally produced APN can also mediate cardioprotection in paracrine and autocrine manners ${ }^{[33,34,36]}$. Guo et al reported that cardiac APN protein expression did not change in STZ-induced diabetic rats, whereas plasma APN level was decreased ${ }^{[37]}$. Conversely, our study provided evidence that APN protein was detectable in control myocytes and total APN level was significantly decreased in ventricular myocytes at 12 weeks post-fistula, which was in parallel with a decrease in serum APN level. Although our results could not differentiate the major cardio-protective roles by circulating APN or by cardiomyocyte produced APN, our data suggests that the protein expression of tissue and systemic APN may be regulated by the same mechanisms. However, the underlying mechanism is as yet unknown. One possible reason is the increased production of tumor necrosis factor- $\alpha$ (TNF- $\alpha$ ). Our preliminary data showed that TNF-a was not detectable 
in control myocytes. However, TNF-a levels were elevated in ventricular myocytes at 12 weeks post-fistula. Also, TNF- $a$ levels were significantly increased in serum when animals were subjected to volume overload ${ }^{[38,39]}$. TNF- $\alpha$ may suppress APN expression via JNK (c-Jun N-terminal kinase)- and protein kinase $\mathrm{C}(\mathrm{PKC})$-dependent signaling pathways ${ }^{[40,41]}$. Moreover, TNF-a inhibits APN expression via TNF receptor type $1^{[42]}$.

APN has been described as achieving these protective effects through receptors, AdipoR1/R2, both of which are expressed in cardiomyocytes ${ }^{[37,43,44]}$. In addition to AdipoR1/R2, T-cadherin has recently been identified as a receptor for hexameric and high-molecular-weight (HMW) forms of $\mathrm{APN}^{[22]}$. Although lacking transmembrane and cytoplasmic domains, T-cadherin may act as co-receptor to transmit APN-mediated metabolic signals via an unidentified signaling pathway, further protecting myocardium against injury ${ }^{[23]}$. Caselli et al reported that the mRNA expression of AdipoR1 was significantly upregulated and myocardial AdipoR2 and T-cadherin mRNA expression did not change in a porcine dilated cardiomyopathy model ${ }^{[45]}$. Also, AdipoR2 protein levels did not change in diabetes mellitus ${ }^{[37,46]}$. In this study, the protein expression of AdipoR1/R2 and T-cadherin were all downregulated in ventricular myocytes following 12 weeks fistula. Adenovirus-mediated APN overexpression significantly prevented the reduction of myocardial expressions of APN receptors after 12 weeks fistula, which dramatically rescued the abnormal myocardial contractile function due to volume overload. However, the precise mechanism for depressing the myocardial expression of AdipoR1/R2 and T-cadherin is as yet unknown. A previous study showed that TNF-a functions as a negative regulator of AdipoR1/R2 expression ${ }^{[36]}$. APN overexpression may inhibit the production of TNF- $\alpha$, further improving the expression of AdipoR1 and AdipoR2 in 12-week fistula cardiomyocytes. Furthermore, some studies suggested that insulin reduces AdipoR1 and AdipoR2 expression via the phosphoinositide 3-kinase/Foxo1-dependent pathway in vivo and in vitro ${ }^{[4,47]}$.

AMPK is a stress-activated protein kinase that mediates glucose uptake and fatty acid oxidation in cardiomyocytes ${ }^{[12,48]}$. APN functions to attenuate cardiac hypertrophy mainly through AMPK-dependent signaling pathways ${ }^{[17]}$. AMPK deficiency exacerbated myocardial hypertrophy and dysfunction $^{[11,49,50]}$. Myocardial AMPK activity was enhanced when subjected to short-term pressure overload and myocardial ischemia $^{[51,52]}$. Although we did not test AMPK activity in the early stage of volume overload, our study showed that AMPK activity was significantly decreased in 12-week fistula myocytes. This observation suggests that decreased AMPK activity is a decompensated mechanism that fails to satisfy cardiac energy demand and maintain energy homeostasis in the heart under volume overload and contributes to myocardial hypertrophy and myocyte abnormalities. This mechanism may be partly contributed to hypoadiponectinemia and the decreased myocardial expression of APN due to volume overload. In accordance with this, adenovirus-mediated APN overexpres- sion increased APN levels in serum and in myocytes after 12 weeks fistula, thus enhancing AMPK activity and restoring myocyte contractile function in 12-week fistula myocytes.

In summary, this study examined the role of APN in the volume overload-induced heart failure. Our results showed that hypoadiponectinemia played a causal role in the progression of heart failure under chronic volume overload. Furthermore, the decreased expression of APN and its receptors in myocytes resulted in myocyte contractile abnormalities via the reduced AMPK phosphorylation. Thus, these observations suggested a potential therapeutic application of APN in the treatment of volume overload-induced cardiac dysfunction.

\section{Acknowledgements}

This study was supported by the Animal Health and Disease Research Funds from Auburn University College of Veterinary Medicine.

\section{Author contribution}

Li-li WANG performed most of the experiments/data analysis and drafted the manuscript; Dori MILLER participated in most of the experiments/data analysis and helped drafting the manuscript; Desiree WANDERS performed some of the Elisa experiments; Gayani NANAYAKKARA and Rajesh AMIN prepared the Ad-APN; Robert JUDD helped in animal injection; Edward E MORRISON and Rajesh AMIN helped designing the experiments; Ju-ming ZHONG designed and conducted all the experiments and finalized the manuscript.

\section{References}

1 Go AS, Mozaffarian D, Roger VL, Benjamin EJ, Berry JD, Borden WB, et al. Heart disease and stroke statistics--2013 update: a report from the American Heart Association. Circulation 2013; 127: e6-e245.

2 Goktepe S, Abilez OJ, Parker KK, Kuhl E. A multiscale model for eccentric and concentric cardiac growth through sarcomerogenesis. J Theoreatical Biol 2010; 265: 433-42.

3 Sciarretta S, Sadoshima J. New insights into the molecular phenotype of eccentric hypertrophy. J Mol Cell Cardiol 2010; 49: 153-6.

4 Melenovsky V, Benes J, Skaroupkova P, Sedmera D, Strnad H, Kolar M, et al. Metabolic characterization of volume overload heart failure due to aorto-caval fistula in rats. Mol Cell Biochem 2011; 354: 83-96.

5 Altin SE, Schulze PC. Metabolism of the right ventricle and the response to hypertrophy and failure. Prog Cardiovasc Dis 2012; 55: 229-33.

6 Wang X, Ren B, Liu S, Sentex E, Tappia PS, Dhalla NS. Characterization of cardiac hypertrophy and heart failure due to volume overload in the rat. J Appl Physiol 2003; 94: 752-63.

7 Ding YF, Brower GL, Zhong Q, Murray D, Holland M, Janicki JS, et al. Defective intracellular $\mathrm{Ca}^{2+}$ homeostasis contributes to myocyte dysfunction during ventricular remodelling induced by chronic volume overload in rats. Clin Exp Pharmacol Physiol 2008; 35: 827-35.

8 Haluzik M, Parizkova J, Haluzik MM. Adiponectin and its role in the obesity-induced insulin resistance and related complications. Physiol Res 2004; 53: 123-9.

9 Fang X, Sweeney G. Mechanisms regulating energy metabolism by adiponectin in obesity and diabetes. Biochem Society Transactions 2006; 34: 798-801.

10 Ding G, Qin Q, He N, Francis-David SC, Hou J, Liu J, et al. Adiponectin 
and its receptors are expressed in adult ventricular cardiomyocytes and upregulated by activation of peroxisome proliferator-activated receptor gamma. J Mol Cell Cardiol 2007; 43: 73-84.

11 Shimano M, Ouchi N, Shibata R, Ohashi K, Pimentel DR, Murohara $\mathrm{T}$, et al. Adiponectin deficiency exacerbates cardiac dysfunction following pressure overload through disruption of an AMPK-dependent angiogenic response. J Mol Cell Cardiol 2010; 49: 210-20.

12 Palanivel R, Fang X, Park M, Eguchi M, Pallan S, De Girolamo S, et al. Globular and full-length forms of adiponectin mediate specific changes in glucose and fatty acid uptake and metabolism in cardiomyocytes. Cardiovasc Res 2007; 75: 148-57.

13 Kanda T, Saegusa S, Takahashi T, Sumino H, Morimoto S, Nakahashi $\mathrm{T}$, et al. Reduced-energy diet improves survival of obese KKAy mice with viral myocarditis: induction of cardiac adiponectin expression. Int J Cardiol 2007; 119: 310-8.

14 Youn JC, Kim C, Park S, Lee SH, Kang SM, Choi D, et al. Adiponectin and progression of arterial stiffness in hypertensive patients. Int J Cardiol 2013; 163: 316-9.

15 Teijeira-Fernandez E, Eiras S, Grigorian-Shamagian L, Fernandez A, Adrio B, Gonzalez-Juanatey JR. Epicardial adipose tissue expression of adiponectin is lower in patients with hypertension. J Human Hypertension 2008; 22: 856-63.

16 Hashimoto N, Kanda J, Nakamura T, Horie A, Kurosawa H, Hashimoto $\mathrm{T}$, et al. Association of hypoadiponectinemia in men with early onset of coronary heart disease and multiple coronary artery stenoses. Metabolism 2006; 55: 1653-7.

17 Shibata R, Ouchi N, Ito M, Kihara S, Shiojima I, Pimentel DR, et al. Adiponectin-mediated modulation of hypertrophic signals in the heart. Nat Med 2004; 10: 1384-9.

18 Shibata R, Sato K, Pimentel DR, Takemura Y, Kihara S, Ohashi K, et al. Adiponectin protects against myocardial ischemia-reperfusion injury through AMPK- and COX-2-dependent mechanisms. Nat Med 2005; 11: 1096-103.

19 Shibata R, Izumiya Y, Sato K, Papanicolaou K, Kihara S, Colucci WS, et al. Adiponectin protects against the development of systolic dysfunction following myocardial infarction. J Mol Cell Cardiol 2007; 42: 1065-74.

20 Ding Y, Zou R, Judd RL, Zhong J. Endothelin-1 receptor blockade prevented the electrophysiological dysfunction in cardiac myocytes of streptozotocin-induced diabetic rats. Endocrine 2006; 30: 121-7.

21 Yamauchi T, Kamon J, Ito Y, Tsuchida A, Yokomizo T, Kita S, et al. Cloning of adiponectin receptors that mediate antidiabetic metabolic effects. Nature 2003; 423: 762-9.

22 Hug C, Wang J, Ahmad NS, Bogan JS, Tsao TS, Lodish HF. T-cadherin is a receptor for hexameric and high-molecular-weight forms of Acrp30/adiponectin. Proc Natl Acad Sci U S A 2004; 101: 10308-13.

23 Denzel MS, Scimia MC, Zumstein PM, Walsh K, Ruiz-Lozano P, Ranscht B. T-cadherin is critical for adiponectin-mediated cardioprotection in mice. J Clin Invest 2010; 120: 4342-52.

24 Yan W, Zhang H, Liu P, Wang H, Liu J, Gao C, et al. Impaired mitochondrial biogenesis due to dysfunctional adiponectin-AMPKPGC-1alpha signaling contributing to increased vulnerability in diabetic heart. Basic Res Cardiol 2013; 108: 329.

25 Kehat I, Molkentin JD. Molecular pathways underlying cardiac remodeling during pathophysiological stimulation. Circulation 2010; 122: 2727-35.

26 Rohini A, Agrawal N, Koyani CN, Singh R. Molecular targets and regulators of cardiac hypertrophy. Pharmacol Res 2010; 61: 269-80.

27 Toischer K, Rokita AG, Unsold B, Zhu W, Kararigas G, Sossalla S, et al. Differential cardiac remodeling in preload versus afterload. Circulation 2010; 122: 993-1003.
28 O'Shea KM, Chess DJ, Khairallah RJ, Rastogi S, Hecker PA, Sabbah $\mathrm{HN}$, et al. Effects of adiponectin deficiency on structural and metabolic remodeling in mice subjected to pressure overload. Am J Physiol Heart Circ Physiol 2010; 298: H1639-45.

29 Lee B, Shao J. Adiponectin and energy homeostasis. Rev Endocr Metab Disord 2014; 15: 149-56.

30 Arita Y, Kihara S, Ouchi N, Takahashi M, Maeda K, Miyagawa J, et al. Paradoxical decrease of an adipose-specific protein, adiponectin, in obesity. Biochem Biophys Res Commun 1999; 257: 79-83.

31 Hotta K, Funahashi T, Arita Y, Takahashi M, Matsuda M, Okamoto $Y$, et al. Plasma concentrations of a novel, adipose-specific protein, adiponectin, in type 2 diabetic patients. Arteriosclerosis Thromb Vasc Biol 2000; 20: 1595-9.

32 Sam F, Duhaney TA, Sato K, Wilson RM, Ohashi K, Sono-Romanelli S, et al. Adiponectin deficiency, diastolic dysfunction, and diastolic heart failure. Endocrinology 2010; 151: 322-31.

33 Wang Y, Lau WB, Gao E, Tao L, Yuan Y, Li R, et al. Cardiomyocytederived adiponectin is biologically active in protecting against myocardial ischemia-reperfusion injury. Am J Physiol Endocrinol Metab 2010; 298: E663-70.

34 Dadson K, Liu Y, Sweeney G. Adiponectin action: a combination of endocrine and autocrine/paracrine effects. Frontiers Endocrinol 2011; 2: 62.

35 Shibata R, Sato K, Kumada M, Izumiya Y, Sonoda M, Kihara S, et al. Adiponectin accumulates in myocardial tissue that has been damaged by ischemia-reperfusion injury via leakage from the vascular compartment. Cardiovasc Res 2007; 74: 471-9.

36 Fujioka D, Kawabata K, Saito Y, Kobayashi T, Nakamura T, Takano H, et al. Role of adiponectin receptors in endothelin-induced cellular hypertrophy in cultured cardiomyocytes and their expression in infarcted heart. Am J Physiol Heart Circ Physiol 2006; 290: H240916.

37 Guo Z, Xia Z, Yuen VG, McNeill JH. Cardiac expression of adiponectin and its receptors in streptozotocin-induced diabetic rats. Metabolism 2007; 56: 1363-71.

38 Jobe $\sqcup$, Melendez GC, Levick SP, Du Y, Brower GL, Janicki JS. TNFalpha inhibition attenuates adverse myocardial remodeling in a rat model of volume overload. Am J Physiol Heart Circ Physiol 2009; 297: H1462-8.

39 Kapadia SR, Yakoob K, Nader S, Thomas JD, Mann DL, Griffin BP. Elevated circulating levels of serum tumor necrosis factor-alpha in patients with hemodynamically significant pressure and volume overload. J Am Coll Cardiol 2000; 36: 208-12.

40 Kim KY, Kim JK, Jeon JH, Yoon SR, Choi I, Yang Y. c-Jun N-terminal kinase is involved in the suppression of adiponectin expression by TNF-alpha in 3T3-L1 adipocytes. Biochem Biophys Res Commun 2005; 327: 460-7.

41 Lim JY, Kim WH, Park SI. G06976 prevents TNF-alpha-induced suppression of adiponectin expression in 3T3-L1 adipocytes: putative involvement of protein kinase C. FEBS Lett 2008; 582: 3473-8.

42 Wang Y, Zhao J, Zhang Y, Lau WB, Jiao LY, Liu B, et al. Differential regulation of TNF receptor 1 and receptor 2 in adiponectin expression following myocardial ischemia. Int J Cardiol 2013; 168: 2201-6.

43 Pineiro R, Iglesias MJ, Gallego R, Raghay K, Eiras S, Rubio J, et al. Adiponectin is synthesized and secreted by human and murine cardiomyocytes. FEBS Lett 2005; 579: 5163-9.

44 Yamauchi T, Nio Y, Maki T, Kobayashi M, Takazawa T, Iwabu M, et al. Targeted disruption of AdipoR1 and AdipoR2 causes abrogation of adiponectin binding and metabolic actions. Nat Med 2007; 13: 332-9.

45 Caselli C, Cantinotti M, Del Ry S, Cabiati M, Prescimone T, Storti S, 
et al. Adiponectin plasma levels decrease after surgery in pediatric patients with congenital heart disease. Clin Biochem 2012; 45: 1510-2.

46 Ma Y, Liu Y, Liu S, Qu Y, Wang R, Xia C, et al. Dynamic alteration of adiponectin/adiponectin receptor expression and its impact on myocardial ischemia/reperfusion in type 1 diabetic mice. Am J Physiol Endocrinol Metab 2011; 301: E447-55.

47 Tsuchida A, Yamauchi T, Ito Y, Hada Y, Maki T, Takekawa S, et al. Insulin/Foxo1 pathway regulates expression levels of adiponectin receptors and adiponectin sensitivity. J Biol Chem 2004; 279: 30817-22.

48 Li J, Hu X, Selvakumar P, Russell RRr, Cushman SW, Holman GD, et al. Role of the nitric oxide pathway in AMPK-mediated glucose uptake and GLUT4 translocation in heart muscle. Am J Physiol Endocrinol Metab 2004; 287: E834-41.
49 Wang X, Oka T, Chow FL, Cooper SB, Odenbach J, Lopaschuk GD, et al. Tumor necrosis factor-alpha-converting enzyme is a key regulator of agonist-induced cardiac hypertrophy and fibrosis. Hypertension 2009; 54: 575-82.

50 Konishi M, Haraguchi G, Ohigashi H, Ishihara T, Saito K, Nakano Y, et al. Adiponectin protects against doxorubicin-induced cardiomyopathy by anti-apoptotic effects through AMPK up-regulation. Cardiovasc Res 2011; 89: 309-19.

51 Liao Y, Takashima S, Maeda N, Ouchi N, Komamura K, Shimomura I, et al. Exacerbation of heart failure in adiponectin-deficient mice due to impaired regulation of AMPK and glucose metabolism. Cardiovasc Res 2005; 67: 705-13.

52 Altarejos JY, Taniguchi M, Clanachan AS, Lopaschuk GD. Myocardial ischemia differentially regulates LKB1 and an alternate 5'-AMPactivated protein kinase kinase. J Biol Chem 2005; 280: 183-90. 